\title{
Blockchain in Healthcare
}

\author{
Guangjian Huang*, Abdullah Al Foysal
}

Department of Computer Science and Technology, School of information engineering, Jiangxi University of Science and Technology, Ganzhou, China

Email: *zuidengke@163.com, niloyhasanfoysal440@gmail.com

How to cite this paper: Huang, G. J., \& Foysal, A. A. (2021). Blockchain in Healthcare. Technology and Investment, 12, 168-181. https://doi.org/10.4236/ti.2021.123010

Received: July 9, 2021

Accepted: July 27, 2021

Published: July 30, 2021

Copyright () 2021 by author(s) and Scientific Research Publishing Inc. This work is licensed under the Creative Commons Attribution International License (CC BY 4.0).

http://creativecommons.org/licenses/by/4.0/

\begin{abstract}
The fourth industrial revolution, which will alter the globe, is commonly referred to as Blockchain technology. Blockchain technology provides a decentralized, distributed, and central authority-free environment. Since Bitcoin launched Blockchain, research has been continuing on non-financial use cases to extend their applicability. Healthcare is an industry with a significant influence on the Blockchain. Healthcare has penetrated the enthusiasm for the changing nature of Blockchain technology. Blockchain is frequently viewed as the most necessary and optimal healthcare technology to handle sophisticated and complex security and interoperability concerns. More significantly, the "value" and trust-based system's smart contract mechanism can offer automatic action and reaction. Healthcare, on the other hand, is a complex system. In this paper, we introduce the blockchain and its properties, as well as the significance of the blockchain in healthcare. It also provides blockchain administration, adjudication of claims, interoperability, and application. While in several situations, we observed blockchain technology, the use of blockchain in health care was highly addressed in this paper and the reason why blockchain should be utilized. We introduce the advantages of blockchain as well. Furthermore, we examined the difficulties and prospects for the future and how they may be implemented in more healthcare industries. The paper also discusses the current level of Blockchain application development for healthcare and its limits and topics for further research. This paper aims to demonstrate how Blockchain technologies may be utilized in healthcare and what problems this technology may face in the future and what the Blockchain's prospects are.
\end{abstract}

\section{Keywords}

Blockchain, Healthcare Application, Hospital System, Future Perspectives

\section{Introduction}

The real benefit of interoperability might be unlocked through a Blockchain- 
driven health information exchange. Current middlemen friction and expenses might be reduced or eliminated with Blockchain-based systems. The Precision Medicine Initiative, Patient Care and Outcomes Research (PCOR), and the Nationwide Interoperability Roadmap are all compelling use cases for Blockchain technology. Not only that, but Blockchain technology also poses significant medical problems in terms of reproducibility, data sharing, personal data privacy issues, and patient participation in clinical trials. In the domain of the internet, the Blockchain is bringing the Internet closer to its ultimate goal of decentralization. It is expected that significant consequences for the Blockchain's health will occur. For health care and information technology, this is an exciting time. Health care is seeing an innovative approach to illness prevention as a result of advancements in genetic research and clinical research. While Blockchain technology is not a silver bullet for data standards or system integration problems, it does provide a potential new distributed framework for amplifying and supporting the integration of health care data across multiple applications and stakeholders. It solves a number of problems while also making the system more efficient, decentralized, and safe. Blockchain technology is a game-changing innovation. As a result, a substantial clinical transformation is required to effectively employ technology for the greatest outcome that can be examined and analyzed from a socio-technical standpoint. As technology improves, we will be able to use it more effectively. It is critical to consider the socio-technical issues and solutions for Blockchain. In order to get the greatest results in healthcare, technology is used (Linn \& Koo, 2016).

\subsection{Definition of Blockchain}

A Blockchain is a type of database that is unique. The method that stores information differs from that of a typical database; Blockchains store data in blocks that are then linked together. A new block is added to it whenever fresh data is input. Blockchain Technology is a huge network of people who may function as validators to achieve an agreement on many topics, such as transactions. This technique is verified and utilized for network protection via mathematical verification (Hölbl, Kompara, Kamišalić, \& Zlatolas, 2018).

\subsection{Blockchain Working Process}

In a nutshell, Blockchain technology is distributed ledger technology (DLT) that allows data to be kept globally on thousands of computers while allowing anybody on the network to see any other entries in near real-time. It is built on a peer-to-peer (P2P) architecture. This makes it hard for a user to operate the network or game.

Unconfident members are required to establish agreement on the dispersed nature of the network. Blockchain can have consensus on "rules" (for instance, the number of bitcoins in the package award, mining difficulties, etc.) or on the history of "transacts" (for example, which transactions are permitted and which are not allowed) (that allows determining who owns what). The decentralized 
transaction consensus controls the updating of the leader by passing its duties to the local nodes, which check and add them to the cumulative calculation process independently (longest chain rule) (Tasca \& Tessone, 2017).

\subsection{The Importance of Blockchain in Healthcare}

The lack of a central administrator is one of the key features that distinguish the Blockchain as a cutting-edge healthcare technology. This is due to the fact that the database is still seen as a physical object made up of bits and bytes. And, because the data is a tangible item, the chances of it being lost, misused, or accidentally deleted from manual records are extremely high. Have you ever considered what would happen if the data you have saved in your system's physical memory was damaged by those who have access to it? The security of network infrastructure at all levels is one of the most significant problems for Blockchain healthcare applications. The identities of all participants are checked and validated. Access to electronic health records requires authorization in a consistent manner.

Implementing Blockchain technology, on the other hand, would eliminate the need for a central administrator because cryptography would replace the former's duties. Because the healthcare sector relies on rapid access to a patient's data while still keeping the information private and secret, the Blockchain may reorganize this medical database and allow for secure dissemination. So, you have scalability, data confidentiality, and quick access all in one system. Though the introduction of Blockchain technology to the healthcare industry may be disruptive at times, it will never be an elixir to solve database problems. Instead, it may be an incredible adventure that involves the gradual deployment of Blockchain. So, Blockchain is essentially a secure transaction database that is shared by all parties that have engaged in the computer network. What the Blockchain does now is that it replaces the requirement for a trusted third-party transaction analyzer by recording all transactions that occur in the network of computers and sending copies of all transactions to each and every node in the network. The Blockchain has the potential to be a game-changer since it decreases or eliminates the necessity of a third-party payment processor, lowering expenses for businesses. At the same time, it provides a transparent and trustworthy channel for non-trusted organizations to deal with, without the need for third-party validation. The Blockchain is still in its early stages of development. A consortium, consisting of around 45 major firms, is currently studying Blockchain technology and its applications. The Blockchain is extremely beneficial to businesses that provide transactional services. Because transactions are required in almost every business, the ramifications of the Blockchain will be enormous (Hölbl, Kompara, Kamišalić, \& Zlatolas, 2018).

\subsection{Benefits of Blockchain Using in Healthcare}

Only approximately 5 - 6 percent of CIOs and less than $12 \%$ of industry leaders have included Blockchain technology in their strategic plans. This is the primary 
reason why understanding the practical uses of Blockchain technology in the healthcare industry is critical. I've put up a list of five key ways that Blockchain might help healthcare in 2019 (Hölbl, Kompara, Kamišalić, \& Zlatolas, 2018).

\subsection{Single and Longitudinal Records of the Patient}

Blockchain might be used to create single patient records that include illness information, test findings, and therapies. This also helps doctors give better treatment to patients by allowing them to employ more advanced procedures (Cyran, 2018).

\subsection{Master Patient Indices}

When dealing with a large database of patients, it's common for records to get lost or jumbled up. Furthermore, depending on the EHR you choose, there might be significant differences in the way databases are recorded, which complicates even the most basic data. All of the records are entered into a single data entry book, or ledger, at the same time. For example, if you look for a person's address, you'll find a number of addresses and various keys, but you'll only get one patient ID (Ivan, 2016).

\subsection{Supply Chain Management}

The use of Blockchain technology in healthcare could aid in the screening of supply-demand cycles. For example, how does a monetary transaction take place? Is the contract that your company has accepted going well? Is there any sort of hold-up? All of these details could be easily tracked with Blockchain (Agbo, Mahmoud, \& Eklund, 2019).

\subsection{Claims Adjudication}

Because Blockchain is a transaction and exchange system based on validation, claims are automatically validated when the system approves the way by which a contract is carried out. Furthermore, because there is no central authority, the odds of frauds and mistakes occurring are little to none (Zhang, Schmidt, White, \& Lenz, 2018).

\subsection{Interoperability}

Blockchain technology makes use of sophisticated APIs to make data storage and EHR interoperability a standardized process. Because the Blockchain network is only disseminated to authorize persons in a safe manner, it eliminates the additional costs associated with data reconciliation.

Aside from the previously mentioned advantages, Blockchain technology has the ability to improve clinical trials, revenue cycle management, medication supply management, and fraud prevention (Zhang, Schmidt, White, \& Lenz, 2018).

\subsection{Blockchain Application}

Before we can talk about Blockchain applications, we must first define what an application is. A smart appliance is an internet-connected gadget that provides 
you with more information and control than previously. A code attached to your appliance, for example, can be linked to the internet and inform you when your cookies are ready or if your washing has stopped. These notifications maintain your appliances in excellent working order, save you money on energy, and let you to operate your gadgets when you're away from home, among other things. Encrypting these gadgets on the Blockchain ensures their ownership and allows them to be transferred. There are several Blockchain applications. However, there have some applications that are used in society. They are "asset management: trade processing and settlement, smart appliance insurance: claims processing, smart appliances, payments: cross-border payments, Blockchain government, unconventional money lenders/hard money lending, public value/community, your car/smartphone, vested responsibility, Blockchain internet-of-things (IoT), Blockchain identity, smart appliances, passports, smart appliances, birth, wedding and death certificate, Personal identification". The technological limitation of the unfair use of online surveillance, censorship and the abuse of rights, facilitated by the increasing dependence upon some entities that control access to information online, might also have a role to play in its core operational principles, such as decentralization, transparency, equal opportunities and accountability (Al-Saqaf \& Seidler, 2017).

\section{Reason and Sector of Using Blockchain}

Recently, more and more researchers have devoted to field of Blockchain. it can be applying to lots of applications. In (Brodersen, 2016), researchers show in their paper that a Blockchain gives the security to the party is being using and saves from the third party. As far its uses in social sights and in society but it shows how integrating current health IT investments with a permissioned Blockchain distributed ledger technology (DLT) environment might drive better patient outcomes and align with the ONC's roadmap for change. This technology has many issues with IT paradigms that involve security (specifically data integrity) and privacy, immutably assuring expressed identities, creating highly robust audit trails and improving healthcare-related security for both providers and patients. The creation of secured, trusted, portable healthcare records has a high degree of integrity and fidelity. ONC has identified in its Interoperability Roadmap. The technology development and research we have conducted to date demonstrates that, while the Blockchain has many obstacles to overcome, it will have a positive impact in solving healthcare industry challenges (Linn \& Koo, 2016).

Paper (Ekblaw, Azaria, Halamka, \& Lippman, 2016) shows that about the development of fundamental design changes for Electronic Health Records (EHRs). In this paper, they propose Medical Record a novel, decentralized record management system to handle EHRs, using Blockchain technology. Their system gives patients a comprehensive, immutable log and easy access to their medical information across providers and treatment sites. They incentivize medical stakeholders (researchers, public health authorities, etc.) to participate in the network as Blockchain "miners". This provides them with access to aggregate, anonymized 
data as mining rewards, in return for sustaining and securing the network via Proof of Work. The purpose of this paper is to expose, in preparation for field tests, a working prototype through which we analyze and discuss our approach and the potential for Blockchain in health IT and research. There have two figure to understand this paper easily (Wong, Yee, \& Nøhr, 2018).

In (Linn \& Koo, 2016) researchers describe a Blockchain based access-control manager to health records that would advance the industry interoperability challenges expressed in the Office of the National Coordinator for Health Information Technology's (ONC) Shared Nationwide Interoperability Roadmap. Interoperability is also a critical component any infrastructure supporting Patient cantered Outcomes Research (PCOR) and the Precision Medicine Initiative (PMI). A national health IT infrastructure based on Blockchain has far-reaching potential to promote the development of precision medicine, advance medical research and invite patients to be more accountable for their health. Additionally, the technologies for data storage, security and encryption exist and are in use today. This paper borrows heavily from the Massachusetts Institute of Technology's published research on using a public Blockchain to manage and control access to personal data (Al-Saqaf \& Seidler, 2017).

Paper (Stagnaro, 2017) presents several promising use cases for applying Blockchain technology in health care, especially related to interoperability, claims adjudication, supply chain and longitudinal patient care records. The paper summarizes several use cases for employing Blockchain technology in five key data-driven areas. And they are: 1) Longitudinal health care records; 2) Automated health claims adjudication; 3) Interoperability; 4) Online patient access; 5) Supply chain management. The authors explained these five keys with Blockchain. There was also written that if support for Blockchain from the ONC continues, we may see national or regional ONC-sponsored experimental implementations that may soon lead to meaningful advances in new solutions that lower overall health care costs and contribute toward improved patient participation, and even contributions toward health care research. Given the speed of advancement in Blockchain technology, health care professionals would be wise to closely track new Blockchain-related health care applications. They used this to secure their health record and health initiatives (Brodersen, 2016).

In (Agbo, Mahmoud, \& Eklund, 2019) researchers analyze how Blockchain explore in healthcare after Bitcoin. In this paper they explain about the Blockchain use cases in healthcare include the managements of electronic medical records (EMRs), pharmaceutical supply chain, biomedical research and education, remote patient monitoring (RPM), health insurance claims, health data analytics and other potential areas of healthcare applications. This paper also high light that by applying Blockchain to the management of EMRs, patients will be in control of their own health data and be able to decide how they are used (Ekblaw, Azaria, Halamka, \& Lippman, 2016).

The main contribution of (Benchoufi \& Ravaud, 2017) is that they explain the Blockchain technology in healthcare (transparent checkable methodology) in 
some category. They used this technology in clinical research and explained the sharing of data safety mostly, as like (Linn \& Koo, 2016) Clinical trial phase control (Wong, Yee, \& Nøhr, 2018) Privacy by design and data sharing in community driven medicine. And also said why we can trust this technology and how much safe this is. They used this technology in privacy by design and data sharing in community driven medicine, building reliable clinical studies, privacy by design and data sharing in community driven medicine. In these sections they use the Blockchain technology in healthcare application (Linn \& Koo, 2016).

Paper (Krawiec, Housman, White, Filipova, Quarre, Barr, Nesbitt, Fedosova, Killmeyer, Israel, \& Tsai, 2016) develops in response to the Department of Health and Human Services' Office of the National Coordinator for Health Information Technology (ONC) ideation challenges. This paper explains the Use of Blockchain in Health IT and Health-Related Research. Especially in this paper they said the importance of Blockchain and the opportunities of it. The main points of this paper are: 1) Establishing a trust network; 2) Cost per transaction; 3) Master Patient Index (MPI); 4) Varying data standards; 5) Limited access to population health data; 6) Inconsistent rules and permissions. And the opportunities are: 1) Distributed framework for patient digital identities; 2) Reduced transaction costs; 3) Disintermediation of trust; 4) Shared data; and 5) smart contracts (Stagnaro, 2017).

The Blockchain can also be applied to healthcare data management (Hölbl, Kompara, Kamišalić, \& Zlatolas, 2018). As like EMR: Electronic Medical Record, EHR: Electronic Health Record, PHR: Personal Health Record. In (Hölbl, Kompara, Kamišalić, \& Zlatolas, 2018) they explained three things perfectly (EMR, EHR, PHR). And also show how it works in healthcare. The reason of this paper is to provide an overview of the potential for Blockchain technology in the healthcare system. The review covers technological topics from storing medical records in Blockchains through patient personal data ownership and mobile apps for patient outreach (Agbo, Mahmoud, \& Eklund, 2019).

In (Dimitrov, 2019) researchers explain about the type of Blockchain and they explain how they can work in healthcare. They also talk about that to transform healthcare the focus should be given to the management of data that could benefit from the potential to connect disparate systems and increase the accuracy of EHRs. There have a lot of benefits in different categories in healthcare. As like other healthcare areas that can benefit from Blockchain technology are provider credentials, medical billing, contracting, medical record exchange, clinical trials, and anti-counterfeiting drugs. With regard to the European Coordination committee of the Radiological and Electromedical and Healthcare IT Industry there are still many possibilities for employing Blockchain in healthcare, like medical billing or anti-counterfeiting drugs (Benchoufi \& Ravaud, 2017).

In Table 1 and Figure 1 we compare the management of patient data records (P.D.M), supply chain proof (S.C.P), revenue cycle management (R.C.M) and privacy. We compared the above papers with each of them, and we also rated the above papers with (P.D.M, S.C.P, R.C.M and PRIVACY) these 4 impotent things. 
Table 1. Overview of above paper's.

\begin{tabular}{|c|c|c|c|c|c|}
\hline Paper no & Paper name & $\begin{array}{l}\text { Patient data } \\
\text { management }\end{array}$ & $\begin{array}{l}\text { Supply chain } \\
\text { proof }\end{array}$ & $\begin{array}{c}\text { Revenue cycle } \\
\text { management }\end{array}$ & Privacy \\
\hline 1. & $\begin{array}{l}\text { Brodersen } \\
\text { et al. }(2016)\end{array}$ & High & High & High & Secure \\
\hline 2. & $\begin{array}{c}\text { Ekblaw } \\
\text { et al. }(2016)\end{array}$ & High & Medium & High & Secure \\
\hline 3. & $\begin{array}{l}\text { Linn } \\
\text { et al. (2016) }\end{array}$ & High & High & High & Secure \\
\hline 4. & Stagnaro (2017) & High & Low & High & Secure \\
\hline 5. & $\begin{array}{l}\text { Agbo } \\
\text { et al. (2019) }\end{array}$ & Medium & Low & High & Secure \\
\hline 6. & $\begin{array}{l}\text { Benchoufi } \\
\text { et al. (2017) }\end{array}$ & Low & Medium & High & Secure \\
\hline 7. & $\begin{array}{l}\text { Krawiec } \\
\text { et al. (2016) }\end{array}$ & Medium & Medium & High & Secure \\
\hline 8. & $\begin{array}{l}\text { Hölbl } \\
\text { et al. (2018) }\end{array}$ & Medium & Medium & High & Secure \\
\hline 9. & Dimitrov (2019) & Low & Medium & High & Secure \\
\hline
\end{tabular}

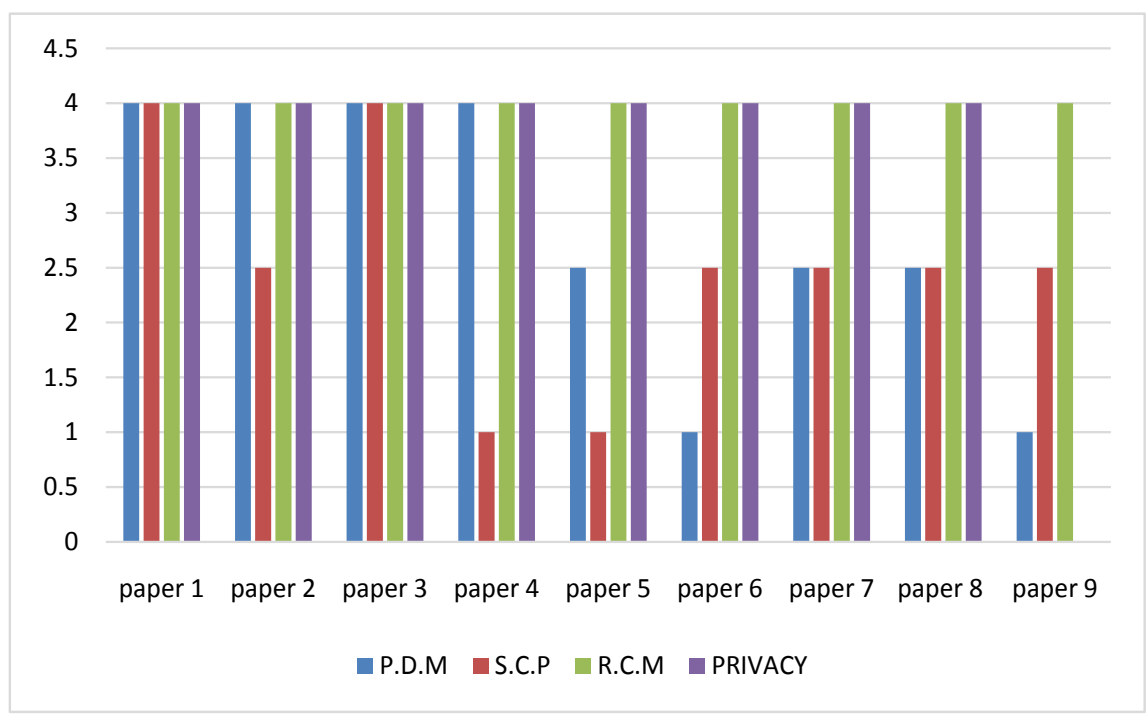

Figure 1. Performance of different papers on P.D.M, S.C.P, R.C.M and PRIVACY.

\subsection{Moving towards a Blockchain-Based Method for Properly Preserving Patient Records}

Modern medical data storage and dissemination strategies have a number of drawbacks that restrict patients' access to their clinical records, impede the availability of critical data to care providers, and, as a result, obstruct the transformation of US healthcare into a learning health system. These flaws might be solved by keeping patient healthcare data in a Blockchain-based storage system. This paper looks at Blockchain as a unique way to secure health data storage, as well as implementation issues and a roadmap for moving from current technologies to a 
Blockchain solution.

A variety of factors contribute to the difficulty of providing and managing access to healthcare information. This includes denying patients and their representatives' access to health-related information. Certain firms regard data analytics as a competitive advantage. While this is legal, it presents substantial and often costly hurdles for patients who need or want to transfer their medical information to another facility. Meaningful Use, the effort credited with the fast adoption of EHRs in the previous seven years, compels providers to allow patients to view, save, and export their data to other sites. Despite the fact that many sources are communicating at least some data with other systems today, indicating minor development in this field, information is still primarily kept in the hands of those who create it. This is such a significant problem that the National Coordinator's Office has devised procedures for identifying and resolving "information blocking" incidents. Due to the challenges of properly sending and distributing health data in a timely manner, the healthcare system suffers.

Developing a Blockchain-based, patient-controlled system for healthcare record transfer and integration might be a solution to these problems. This problem refers to many aspects, such as Blockchain technology goods and services to understand how Blockchain technology can assure the safety and efficiency of huge data storage and sharing in healthcare. Blockchain technology is based on three essential ideas. To begin, data is kept in a publicly accessible, irreversible transaction ledger. Because transactions can't be reversed or modified, there is always a complete and irrefutable record of all transactions. Second, Blockchains are immune to attacks and defects since they are constructed on a network of computer nodes. Thirds, while everyone on the system has access to the data describing each activity, the data stored in the Blockchain is not. The Blockchain is built on pseudo anonymity and public key infrastructure (PKI), which allows the contents of the Blockchain to be secured in a way that is exceedingly difficult to decrypt. When it comes to applying Blockchain technology to medical information, each of these core notions remains true (Ivan, 2016).

\subsection{Blockchain as Platform for Health Information Sharing}

By allowing data exchange between providers and electronic health record (EHR) systems, Blockchain technology has the potential to change healthcare delivery. But major barriers prevent this technology from being generally implemented in the healthcare industry. Two of the main issues related to the use of Blockchains to share information in health care are: securing sensitive health information and deploying and installing Blockchain software in various hospital contexts. We have designed our Blockchain solution with a distributed microwave architecture to enable our system's -core functions to be encapsulated in single services that can be scaled on a specific level based on the needs of a particular hospital system deployment. To ensure easy implementation in a hospital system, We designed essential components for handling cryptographic secrets safely, con- 
necting with Blockchain nodes, simplifying big file sharing, enabling secondary-index based lookups, and integrating external business logic that regulates how users interact with Smart Contracts as part of this architecture (Cyran, 2018).

\subsection{Easy to Use and Implement in a Hospital System}

For our Blockchain technology to be accepted and used in the healthcare system, we need to make the deployment process as simple as possible. Variations in hospital information technology (IT) infrastructure can often stymie rollout and lead to a greater reliance on IT management. The Blockchain solution is wrapped within a specific virtual machine image in our system, which is known as containerization. Containerization ensures our software's portability, simplifies deployment, and lowers maintenance costs across a range of infrastructure setups. Because, all that is required to join a network is one or more computer instances and any IT help needed to connect to an external Blockchain network, an application that can be easily established in any infrastructure setting saves a lot of money. A scalable solution and services within a Blockchain architecture that perform data sharing features such as encryption, decryption, transaction signature facilitation, and storage of cryptographical objects that comply with best security practices are required for easy implementation within a hospital system. The capacity of our technology to provide semantic interoperability within and between hospital systems is a critical component of its deployment. Our approach relies on health data uploads that are triggered by software components contained inside electronic health record (EHR) systems that are part of our Blockchain network as part of the bigger solution. This information is provided by a number of different EHR systems, including Cerner and EPIC. The data is encoded using open standards based on FHIR HL7 in order for all consortium members to be able to compute it, independently of the software they use in their local environment. Permissioned Blockchain capabilities must be supplemented with effective encryption components for integrity, security, and portability of user-owned data to address these concerns and improve the interoperability of health data. In this work, we presented our novel approach for a Blockchain system that allows for the secure flow of data while also ensuring transaction privacy using cryptographic methods. Our approach, which combines strengthened security features with containerization, creates a reliable and simple-to-implement solution that will accelerate adoption of a Blockchain-based health data sharing network (Cyran, 2018).

\subsection{Blockchain Technology Use Cases in Healthcare}

Blockchain technology eliminates the need for a centralized authority to guarantee information integrity and ownership, as well as to mediate transactions and the exchange of digital assets, by allowing safe and pseudo-anonymous transactions and direct agreements between participants. It has crucial qualities like immutability, decentralization, and openness that might help with critical healthcare chal- 
lenges including incomplete records at the point of service and restricted access to patients' own health data. Interoperability allows software apps and technology platforms to connect safely and seamlessly, share data, and utilize that data across health organizations and app suppliers, resulting in a more efficient and effective healthcare system. Due to a lack of interoperability, healthcare today suffers from siloed and fragmented data, delayed communications, and separate workflow tools. In a safe and pseudo-anonymous manner, the Blockchain provides access to longitudinal, full, and tamper-aware medical records that are kept in fragmented systems (Zhang, Walker, White, Schmidt, \& Lenz, 2017).

\subsection{Smart Contracts (Blockchain) in Healthcare: What They Could Be Used for and Why No One Is Using Them}

Today's healthcare organizations face tremendous issues in terms of security, privacy, openness, consent, and data exchange. The proliferation of the Internet of Things (IoT), the implementation of the General Data Protection Regulation (GDPR), the rising trend of patients self-managing their diseases, and patients' eagerness to share their self-collected health data with primary and secondary health organizations all add to the complexity of these issues. Smart contracts, which are based on Blockchain technology, may be a viable solution to these problems. Patients are increasingly involved in controlling their conditions by collecting health data via mobile devices and sensors, in addition to the data collected by healthcare organizations.

Patients are willing to engage, and sharing their self-collected data with medical systems has a good influence on illness treatment. Blockchain technology is gaining a lot of attention in the healthcare industry, and it promises a lot of benefits, including smart healthcare care and customer participation. Smart Blockchain-based contracts, some of them called Blockchain 2.0, are protocols which enable and make irreversible verification and enforcement of legal agreements between two or more parties. But also, three authors said that, the way medical records are stored will not change as a result of Blockchain. Because entering large volumes of medical data, such as computed tomography (CT) scans, would make the Blockchain bloated and impossible to manage, it can only be used as a register. Whether or not Blockchain is employed, the issues of medical data storage remain the same (Giordanengo, 2019).

\subsection{Challenges and Future Perspectives}

Healthcare is a clinical sector with extensive data generation, access to and frequent distribution of a great amount of information. Due to the sensitive nature of data and limiting considerations such as security and privacy, storing and spreading this vast volume of data is both necessary and difficult.

Safe, secure and scalable information (SSS) data exchange is very important for the diagnosis and for integrated clinical decision-making in the healthcare profession and clinical settings. The practice of data sharing is vital if clinics are 
to be able to send their patients' clinical data to the relevant authorities for rapid follow-up. These careers and medical practitioners should be able to transmit their patients' clinical data in a secure and timely way, ensuring that both parties have accurate and up-to-date information about health status. The use of Blockchains to supply safe and safe healthcare data (Agbo, Mahmoud, \& Eklund, 2019; Benchoufi \& Ravaud, 2017), biomedical and e-health data exchange, brain modelling and thinking has attracted considerable attention in recent times. A Blockchain is based on a peer-to-peer (P2P) network. It's essentially a peer-to-peer integrated multi-field network architecture made up of encryption, algorithms, and mathematical expressions targeted at employing distributed consensus techniques to solve traditional distributed database synchronization problems. The Blockchain technology is made up of six main components: Decentralization, transparency, immutability, autonomy, open source, and anonymity are all terms used to describe a system that is decentralized, transparent, and immutable (Siyal, Junejo, Zawish, Ahmed, Khalil, \& Soursou, 2019).

\subsection{Blockchain-Based Decentralized Healthcare Applications Assessment Metrics}

Blockchain is a distributed ledger, thrustless technology that enables safe, pseudo-anonymous transactions by combining transparency, immutability, and consensus features. Smart tracts are constructed on top of a block chain to allow decentralized apps to programming interface with the Blockchain. The interest in health care is driven by programmable Blockchains as a potential solution to critical problems, such as poor communications, wasteful reporting and fragmented health information.

Blockchain is a novel platform that does away with the need for a single, centralized authority while yet allowing for safe and pseudo-anonymous transactions and agreements to be made directly between participants. Through cryptography and game theory, it provides decentralization, immutability, and consensus. Smart contracts are code that may be executed based on predetermined criteria and are constructed on top of a Blockchain. They make it possible to create decentralized apps that interface with Blockchains and store data on the Blockchain (Zhang, Schmidt, White, \& Lenz, 2018; Johnston, Yilmaz, Kandah, Bentenitis, Hashemi, Gross et al., 2014).

\subsection{Some Random Thoughts of Authors}

Researchers looked at current healthcare systems and identified the adjustments that are needed to promote interoperability. Kellermann et al. examined current systems in terms of acceptance, ease of use, and interoperability, and highlighted the adjustments that were required to improve these features. Jones et al. undertook a thorough assessment of health information technology, focusing on its quality, safety, efficiency, and contextual and implementation implications. During the technological lifespan, the important concerns outlined by Cresswell et al 
in implementing the deployment of large-scale health IT for phases including change need, system selection, implementation planning and maintenance and review have been recognized (Kellermann \& Jones, 2013; Jones, Rudin, Perry, \& Shekelle, 2014; Cresswell, Bates, \& Sheikh, 2013).

\section{Conclusion}

The current Blockchain research trends in healthcare were explored in this paper. Because of the sensitive nature of the data being processed and maintained, Blockchain technology is seen as having tremendous potential for application in healthcare. The study's goal was to determine the present state of Blockchain research and implementation in the healthcare industry. However, if the ONC's support for Blockchain continues, we may soon see national or regional ONCsponsored experimental implementations that lead to significant advances in new solutions that reduce overall healthcare costs, improve patient participation, and even contribute to healthcare research. Given the rapid growth of Blockchain technology, health care providers would be prudent to keep a close eye on emerging Blockchain-related health care applications.

\section{Acknowledgements}

I would like to thanks my lecturer Guangjian Huang for his support and help. For his help and guidelines, I could finish this paper properly. The publication of this work was supported by the Jiangxi University of Science and Technology.

\section{Conflicts of Interest}

The authors declare no conflicts of interest regarding the publication of this paper.

\section{References}

Agbo, C. C., Mahmoud, Q., \& Eklund, J. M. (2019). Blockchain Technology in Healthcare: A Systematic Review. Healthcare, 7, 33-56. https://doi.org/10.3390/healthcare7020056

Al-Saqaf, W., \& Seidler, N. (2017). Blockchain Technology for Social Impact: Opportunities and Challenges Ahead. Journal of Cyber Policy, 2, 338-354. https://doi.org/10.1080/23738871.2017.1400084

Benchoufi, M., \& Ravaud, P. (2017). Blockchain Technology for Improving Clinical Research Quality. Trials, 18, 3-35. https://doi.org/10.1186/s13063-017-2035-Z

Brodersen, C. et al. (2016). Blockchain: Securing a New Health Interoperability Experience. Accenture LLP.

Cresswell, K. M., Bates, D. W., \& Sheikh, A. (2013). Ten Key Considerations for the Successful Implementation and Adoption of Large-Scale Health Information Technology. Journal of the American Medical Informatics Association, 20, 9-13. https://doi.org/10.1136/amiajnl-2013-001684

Cyran, M. A. (2018). Blockchain as a Foundation for Sharing Healthcare Data. Blockchain in Healthcare Today, 1, 1-6. https://doi.org/10.30953/bhty.v1.13

Dimitrov, D. V. (2019). Blockchain Applications for Healthcare Data Management. Health- 
care Informatics Research, 25, 51-56. https://doi.org/10.4258/hir.2019.25.1.51

Ekblaw, A., Azaria, A., Halamka, J., \& Lippman, A. (2016). A Case Study for Blockchain in Healthcare: "MedRec" Prototype for Electronic Health Records and Medical Research Data. Proceedings of IEEE Open \& Big Data Conference, Chicago, August 2016, 13.

Giordanengo, A. (2019). Possible Usages of Smart Contracts (Blockchain) in Healthcare and Why No One Is Using Them.

Hölbl, M., Kompara, M., Kamišalić, A., \& Zlatolas, L. N. (2018). A Systematic Review of the Use of Blockchain in Healthcare. Symmetry, 10, 470.

https://doi.org/10.3390/sym10100470

Ivan, D. (2016). Moving toward a Blockchain-Based Method for the Secure Storage of Patient Records. In ONC/NIST Use of Blockchain for Healthcare and Research Workshop (pp. 1-11). ONC/NIST.

Johnston, D., Yilmaz, S. O., Kandah, J., Bentenitis, N., Hashemi, F., Gross, R. et al. (2014). The General Theory of Decentralized Applications, Dapps. GitHub, June 9.

Jones, S. S., Rudin, R. S., Perry, T., \& Shekelle, P. G. (2014). Health Information Technology: An Updated Systematic Review with a Focus on Meaningful Use. Annals of Internal Medicine, 160, 48-54. https://doi.org/10.7326/M13-1531

Kellermann, A. L., \& Jones, S. S. (2013). What It Will Take to Achieve the As-Yet-Unfulfilled Promises of Health Information Technology. Health Affairs, 32, 63-68.

https://doi.org/10.1377/hlthaff.2012.0693

Krawiec, R. J., Housman, D., White, M., Filipova, M., Quarre, F., Barr, D., Nesbitt, A., Fedosova, K., Killmeyer, J., Israel, A., \& Tsai, L. (2016). Blockchain: Opportunities for Health Care.

Linn, L. A., \& Koo, M. B. (2016). Blockchain for Health Data and Its Potential Use in Health It and Health Care Related Research. In ONC/NIST Use of Blockchain for Healthcare and Research Workshop (pp. 1-10). ONC/NIST.

Siyal, A. A., Junejo, A. Z., Zawish, M., Ahmed, K., Khalil, A., \& Soursou, G. (2019). Applications of Blockchain Technology in Medicine and Healthcare: Challenges and Future Perspectives. Cryptography, 3, 3. https://doi.org/10.3390/cryptography3010003

Stagnaro, C. (2017). White Paper: Innovative Blockchain Uses in Health Care. Freed Associates.

Tasca, P., \& Tessone, C. (2017). Taxonomy of Blockchain Technologies. Principles of Identification and Classification. Social Science Electronic Publishing.

Wong, M. C., Yee, K. C., \& Nøhr, C. (2018). Socio-Technical Considerations for the Use of Blockchain Technology in Healthcare. Studies in Health Technology and Informatics, 247, 636-640.

Zhang, P., Schmidt, D. C., White, J., \& Lenz, G. (2018). Blockchain Technology Use Cases in Healthcare. In Advances in Computers (Vol. 111, pp. 1-41). Elsevier. https://doi.org/10.1016/bs.adcom.2018.03.006

Zhang, P., Walker, M. A., White, J., Schmidt, D. C., \& Lenz, G. (2017). Metrics for Assessing Blockchain-Based Healthcare Decentralized Apps. 2017 IEEE 19th International Conference on e-Health Networking, Applications and Services (Healthcom), Vol. 1, 1-8. https://doi.org/10.1109/HealthCom.2017.8210842 